23

\title{
Многооконные акустооптические фильтры для корреляционной спектроскопии
}

\author{
(ㄱ М.М. Мазур ${ }^{1}$, Ю.А. Судденок ${ }^{1}$, В.Э. Пожар ${ }^{1,2}$ \\ ${ }^{1}$ Государственный научный центр ВНИИФТРИ, \\ 141570 Менделеево, Московская обл., Россия \\ ${ }^{2}$ НИЯУ „МИФИ“, \\ 115409 Москва, Россия \\ e-mail: s-mmazur@mail.ru
}

Поступила в редакцию 07.06.2019 г.

В окончательной редакции 07.06.2019 г.

Принята к публикации 05.11.2019 г.

Рассмотрена проблема создания оптических фильтров с произвольной кривой пропускания для задач корреляционной спектроскопии. Экспериментально показано, что акустооптические фильтры с цифровым синтезом управляющего высокочастотного сигнала позволяют создавать функции пропускания разного типа: многооконные, с варьируемым положением и шириной окон, в том числе асимметричной формы, что открывает возможность синтеза функций пропускания сложного вида.

Ключевые слова: акустооптические фильтры, управляемая функция пропускания, корреляционная спектроскопия.

DOI: $10.21883 /$ OS.2020.02.48980.211-19

Оптический спектральный анализ широко используется для определения химического состава образцов, их структуры и состояния. Одним из эффективных и оперативных методов спектрального анализа является корреляционная спектроскопия $[1,2]$, основанная на выявлении сходства спектра пропускания оптического фильтра и спектров поглощения или излучения объекта. Если эти функции „коррелируют“, то регистрируемый спектрометром сигнал значительно превышает уровень сигнала, получаемого при регистрации произвольного, некоррелированного спектра излучения (при равных условиях). Имитируя спектрометром спектральные функции разных веществ, можно определить их наличие в составе образца. Этот метод, хотя и является приблизительным и не может использоваться в качестве универсального, позволяет получать информацию достаточно быстро - без сканирования спектра и подходит для задач реального времени. В некоторых случаях, например, при отсутствии в образце других веществ, чьи спектры накладываются на спектр контролируемого вещества, этот метод может давать вполне надежную информацию. Корреляционная спектроскопия к тому же дает преимущества благодаря одновременной регистрации светового потока в нескольких спектральных окнах, что эквивалентно повышению светосилы спектрометра и позволяет повысить чувствительность прибора и соответственно снизить порог обнаружения веществ или минимальное время регистрации.

Для реализации метода корреляционной спектроскопии необходимо уметь создавать фильтры с заданной формой функции пропускания. Наиболее подходящими технологиями для этого являются перестраиваемые аку- стооптические фильтры [3,4], основанные на эффекте дифракции света на ультразвуковых волнах, динамически создающих в среде структуру с пространственнопериодически изменённым показателем преломления, являющуюся для света фазовой дифракционной решеткой. Такая решетка способна осуществлять селективную дифракцию света, что позволяет создавать на этой основе узкополосные фильтры, перестраиваемые по спектру путем изменения частоты ультразвука. Спектрометры на основе таких акустооптических фильтров представляют собой отдельный класс приборов [4] и могут использоваться для экспресс-анализа образцов во внелабораторных условиях. С учетом того, что такие спектрометры могут перестраиваться на любую длину волны за одно и то же время (без сканирования), для них были разработаны специальные методы анализа, основанные на измерении спектров лишь на нескольких длинах волн, связанных с оптическими свойствами детектируемых веществ или элементов (многоволновые методы [5] и фрагментарная спектральная регистрация [6]).

В ряде задач, таких как эмиссионная спектроскопия, спектроскопия комбинационного рассеяния, абсорбционная спектроскопия газов, линии отдельных веществ практически не перекрываются, а потому спектральный образ веществ представляет собой набор отдельных узких линий. В этом случае эквивалентную (коррелированную) спектральную функцию пропускания можно создать с помощью АО фильтров несколькими способами, например, путем многочастотного возбуждения ультразвука [7] или путем соответствующей вариации частоты ультразвука [8]. В более общем случае линии могут образовывать скопления, группы и тогда в спектре 

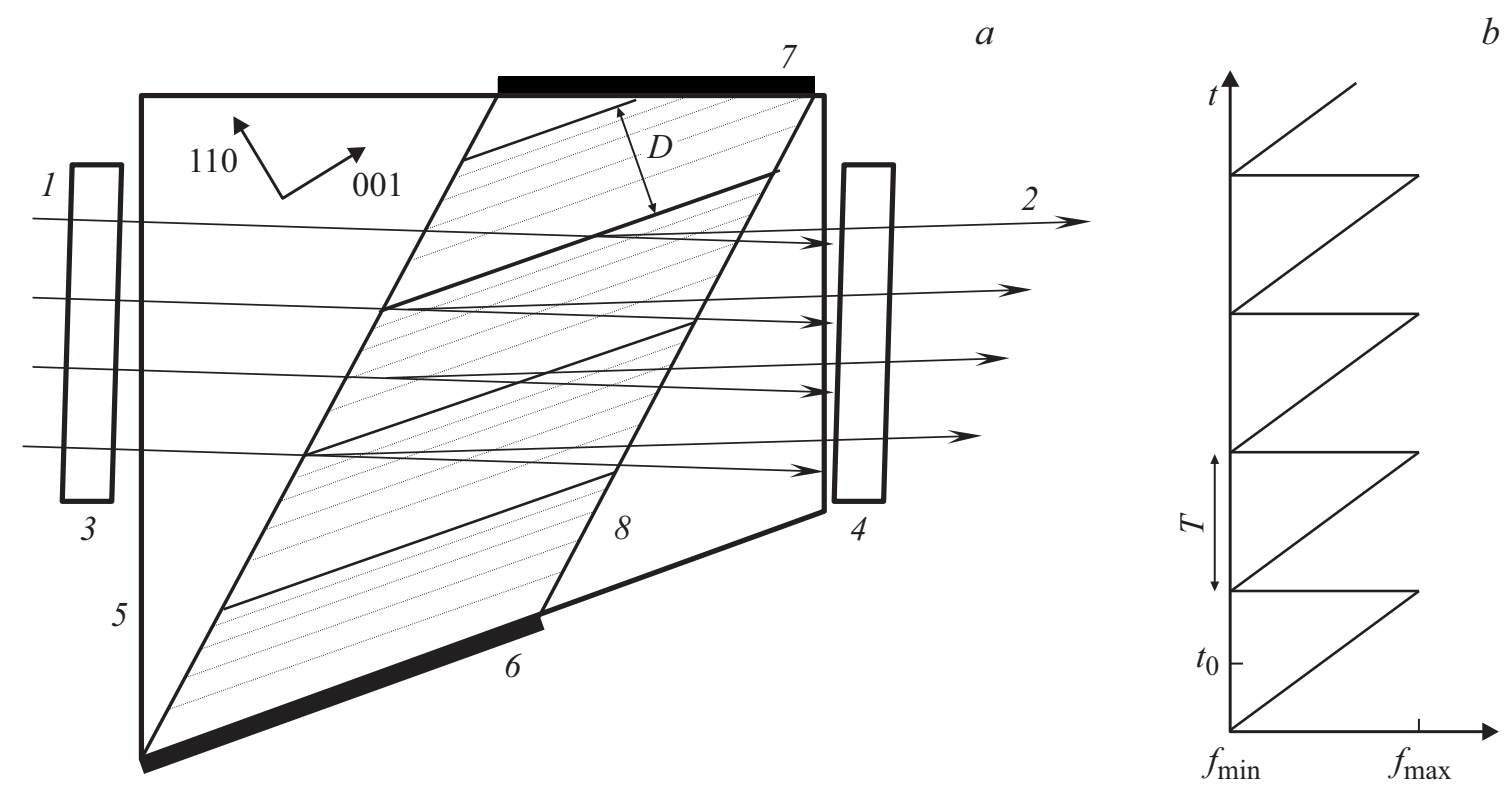

Рис. 1. Схема дифракции света пучка на ЛЧМ ультразвуковой волне $(a)$ и график модуляции частоты $(b)$. $1,2-$ падающий и дифрагированный световые пучки, 3, 4 - скрещенные поляризаторы. 5 - АО кристалл, 6, 7 - акустический излучатель и поглотитель, $8-$ акустический пучок. Стрелки вверху обозначают ориентацию осей АО кристалла. $D-$ пространственный период модуляции решетки.

появляются полосы различной ширины. Поэтому актуальной является задача создания многооконной функции с заданным положением окон пропускания и с различной шириной окон.

Задача расширения полосы пропускания АО фильтров решалась многими авторами в разные периоды и с использованием разной техники $[9,10]$. Различные особенности инструментальных (аппаратных) функций АОФ при частотной модуляции ультразвуковой волны исследовались ранее в работах [11-14]. Все методы основаны на быстром непрерывном изменении частоты, подаваемой на пьезопреобразователь АО фильтра таком, что на пути падающего тонкого оптического пучка встречаются участки решетки с разным периодом и на каждом таком участке дифрагирует собственная спектральная компонента света, благодаря чему и расширяется полоса пропускания АО фильтра. Таким образом, комбинация этих двух подходов, мультичастотного и частотно-модулированного, дает возможность решить рассматриваемую задачу.

В работе описаны особенности реализации этой технологии, приведены примеры получаемых функций пропускания, обсуждается связь различных характеристик такого АО фильтра.

Принципиальная схема фильтрации света представлена на рис. 1. Линейно-поляризованный на входе световой пучок падает на ультразвуковую волну, возбуждаемую в кристалле с помощью акустического излучателя. Вследствие упруго-оптического эффекта акустическая волна вызывает локальные изменения показателей преломления света одноосного АО кристалла и в области распространения волны образуется динамическая фазовая брэгговская решетка, вызывающая дифракцию одной спектральной составляющей света с изменением направления распространения и направления поляризации на ортогональную. Лишь эта спектральная компонента проходит через выходной поляризатор, скрещенный со входным, и это обеспечивает селективную фильтрацию света. Длина волны $\lambda$ прошедшего излучения однозначно связана с периодом ультразвуковой решетки, и соответственно с частотой ультразвука $f: \lambda \sim 1 / f$. Таким образом, перестраивая частоту электрического сигнала, подаваемого на излучатель, можно изменять положение полосы пропускания АО фильтра.

Акустооптическая кристаллическая ячейка на рис. 1 имеет достаточно сложную форму с непараллельными гранями, что связано, во-первых, с отклонением светового пучка при дифракции, а во-вторых, со значительным сносом ультразвукового пучка, вызываемого сильной анизотропией акустических свойств АО кристаллов. Ориентация оптической оси кристалла [001] определяется в результате оптимизации параметров АО фильтра, в частности эффективности дифракции. Акустический поглотитель препятствует образованию отраженных волн, которые могли бы создать дополнительные брэгговские решетки, вызывая паразитную дифракцию.

В соответствии с рассматриваемой задачей частота управляющего модулированного сигнала, подаваемого на АО ячейку, не является постоянной, быстро меняясь во времени, например, пилообразным образом:

$$
f(t)=f_{0}+f^{\prime}\left(t-t_{0}\right), \quad t_{0}-T / 2<t<t_{0}+T / 2 .
$$

Оптимальным периодом модуляции является такой, при котором каждый парциальный луч падающего све- 

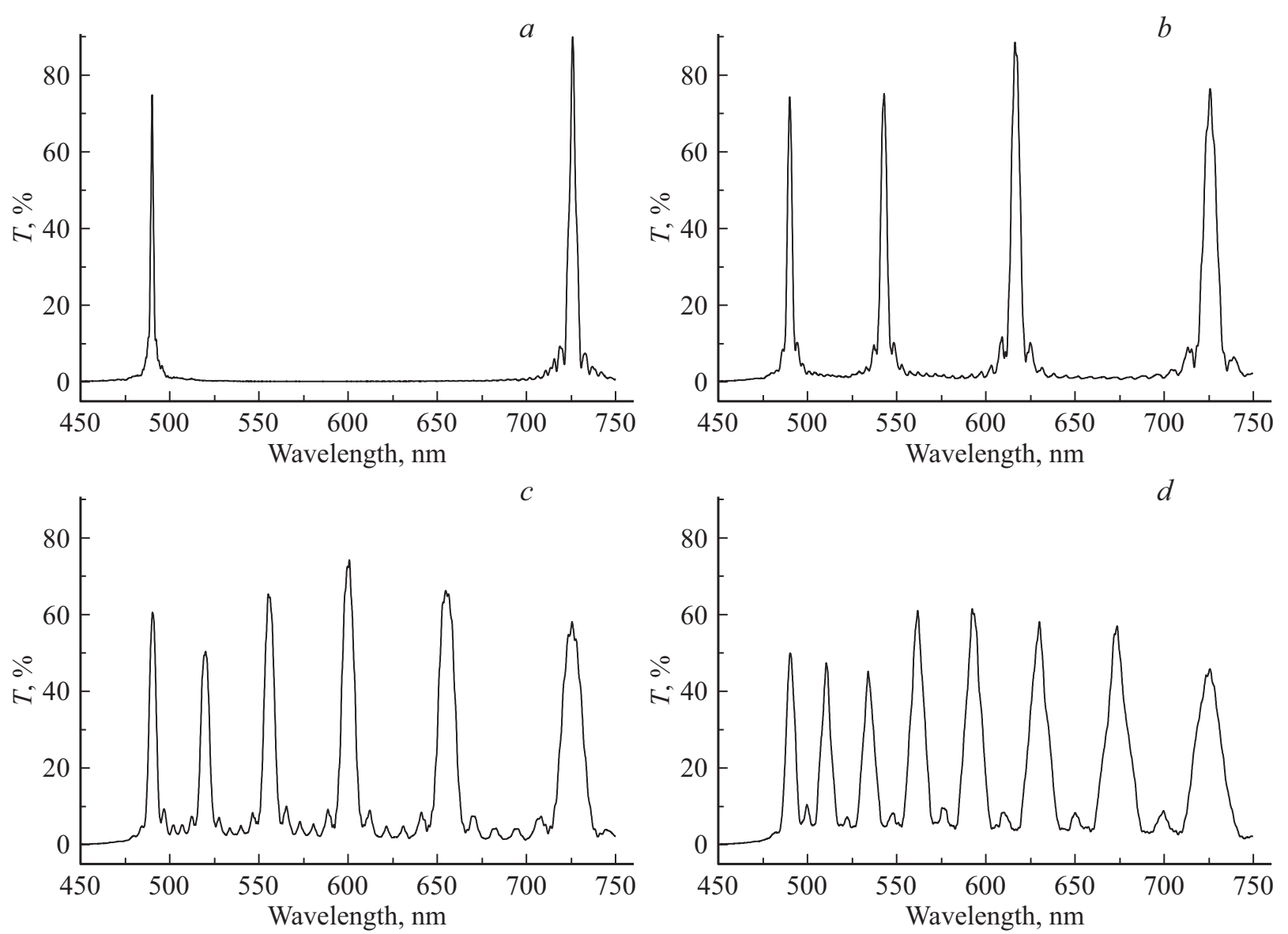

Рис. 2. Вид „многооконных“ аппаратных функций АО фильтра, образованных путем разбиения интервала девиации частоты на соответствующее число $2(a), 4(b), 6(c), 8(d)$ участков с разными частотами.

тового потока проходит через все частотные компоненты частотно-модулированной ультразвуковой волны. Использование линейной пилообразной модуляции (1) дает возможность равномерного уширения окна пропускания $[11,15]$. Для профилирования этой функции временная зависимость частоты подбирается соответствующим образом. С учетом того, что характерное время накопления сигнала (миллисекунды), как правило, на много порядков превосходит период повторения частотного распределения (микросекунды), регистрируемая функция пропускания усредняется по многим периодам повторения распределения. Кроме того, имеет место и усреднение по сечению падающего светового пучка, а потому форма окна пропускания повторяет распределение частоты ультразвука и фактически не зависит от времени.

Оптический фильтр характеризуется несколькими спектральными параметрами, в частности рабочим диапазоном $\left(\lambda_{\min }, \lambda_{\max }\right)$, составляющим обычно не более октавы $\lambda_{\max } / \lambda_{\min }<2$, и полосой пропускания $\Delta \lambda$, определяемой длиной $\mathrm{AO}$ взаимодействия $\Delta \lambda \sim \lambda^{2} / L$. С учетом соотношения $\Delta \lambda \ll\left(\lambda_{\max }-\lambda_{\min }\right)$ в пределах рабочего диапазона можно создать много окон (рис. 2).
Предельное число окон $N_{\max }$ определяется двумя факторами. Во-первых, при увеличении числа полос $N$ на фиксированном по длине участке кристалла должно последовательно помещаться $N$ разных частот, так что эффективная длина взаимодействия для каждого участка пропорционально уменьшается $L_{\mathrm{eff}}(N)=L / N$, а ширина окон - увеличивается $\Delta \lambda(N) \sim 1 / L_{\mathrm{eff}}=N / L$. Во-вторых, при предельном сближении окон возникает эффект биения двух близких частот, что порождает нежелательную временную нестационарность функции пропускания. Поэтому минимально приемлемое расстояние между пиками должно в несколько раз $(G)$ превосходить ширину соответствующих пиков: $\left(\lambda_{m+1}-\lambda_{m}\right) / \Delta \lambda_{m}(N) \geq G$. При разделении всего рабочего диапазона оптических частот на $N$ участков и с учетом спектральной зависимости ширины окон $\Delta \lambda_{m} \sim\left(\lambda_{m}\right)^{2}$ предельное количество окон можно приблизительно оценить по следующей формуле:

$$
\begin{aligned}
N_{\max } & =\left[\left(1 / \lambda_{\min }-1 / \lambda_{\max }\right) /(G \Delta v)\right]^{1 / 2} \\
& \equiv\left[\left(f_{\max }-f_{\min }\right) /(G \Delta f)\right]^{1 / 2},
\end{aligned}
$$

где $\Delta v=\lambda^{2} \Delta \lambda=$ const - ширина окна АО фильтра без модуляции, выраженная в единицах пространственных 

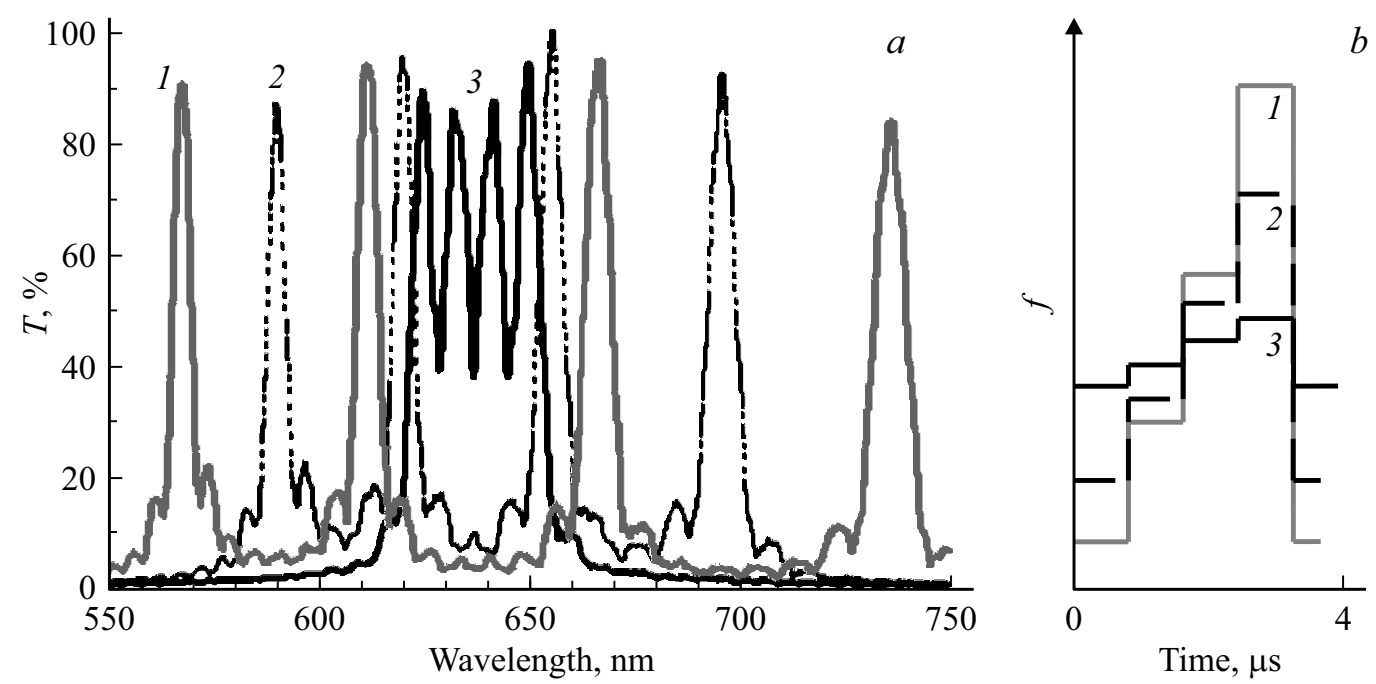

Рис. 3. Управление расстоянием между окнами: $a-$ формы функций пропускания, $b-$ временные диаграммы.
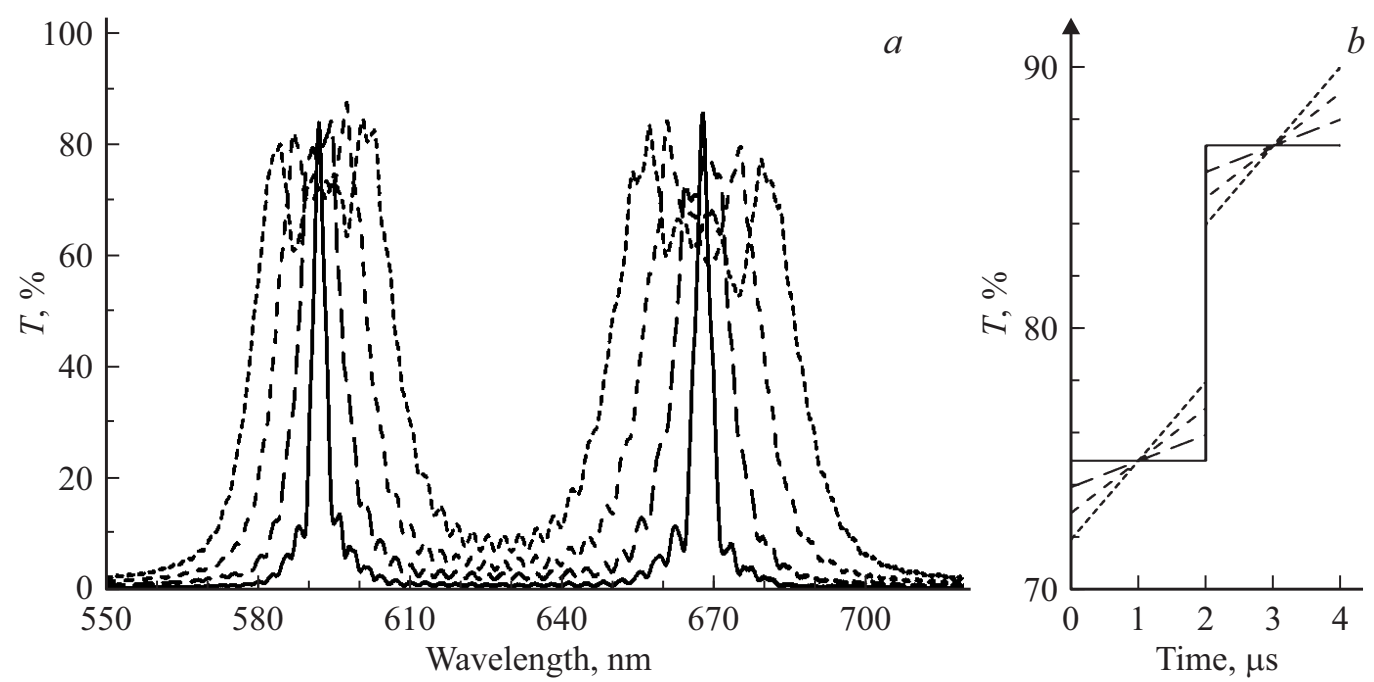

Рис. 4. Управление шириной двух окон функции пропускания $(a)$ путем изменения скорости линейной частотной модуляции ультразвука $(b)$.

оптических частот $v\left(\mathrm{~cm}^{-1}\right), f_{\max }, f_{\min }-$ диапазон подаваемых на АО фильтр управляющих радиочастот, соответствующий рабочему спектральному диапазону, $\Delta f$ - ширина окна АО фильтра, выраженная в единицах радиочастот $(\mathrm{MHz})$. Для использованного в работе АО фильтра эти величины составляли $f_{\max }=122 \mathrm{MHz}$ $\left(\lambda_{\min } \approx 450 \mathrm{~nm}\right), f_{\min }=65 \mathrm{MHz}\left(\lambda_{\min } \approx 750 \mathrm{~nm}\right), \Delta f=$ $=0.2 \mathrm{MHz}(\Delta \lambda \approx 1.5 \mathrm{~nm}$ при $\lambda \approx 450 \mathrm{~nm})$, так что при $G=3$ получаем $N \approx 10$.

Для формирования функций пропускания произвольного вида, необходимых в задачах корреляционной спектроскопии, в основном достаточно уметь синтезировать многооконную функцию пропускания с заданным положением окон, с различной их шириной и с асимметричным профилем. Ниже приведены примеры синтеза функций пропускания $\mathrm{AO}$ фильтра с этими особенностями.
На рис. $3, a$ продемонстрирована возможность создания четырехоконной функции пропускания с регулируемым расстоянием между пиками. В этом примере окна расположены эквидистантно, но, очевидно, что аналогично их можно расположить произвольно в пределах диапазона. Как видно, при сближении на расстояние, сравнимое с шириной окна, функция пропускания образует единую полосу. При $G \approx 5$ (график 2 ) соседние окна практически не влияют на форму друг друга. Временные зависимости сигнала, формирующие эти кривые (рис. $3, b)$, сформированы из интервалов с фиксированными частотами и не используют модуляции.

Возможность управления шириной окон функции пропускания показана на рис. 4, $a$ на примере двух фиксированных окон. В этом примере ширина окон меняется за счет изменения величины девиации частоты (рис. $4, b$ ). Как видно, при значительном расширении 

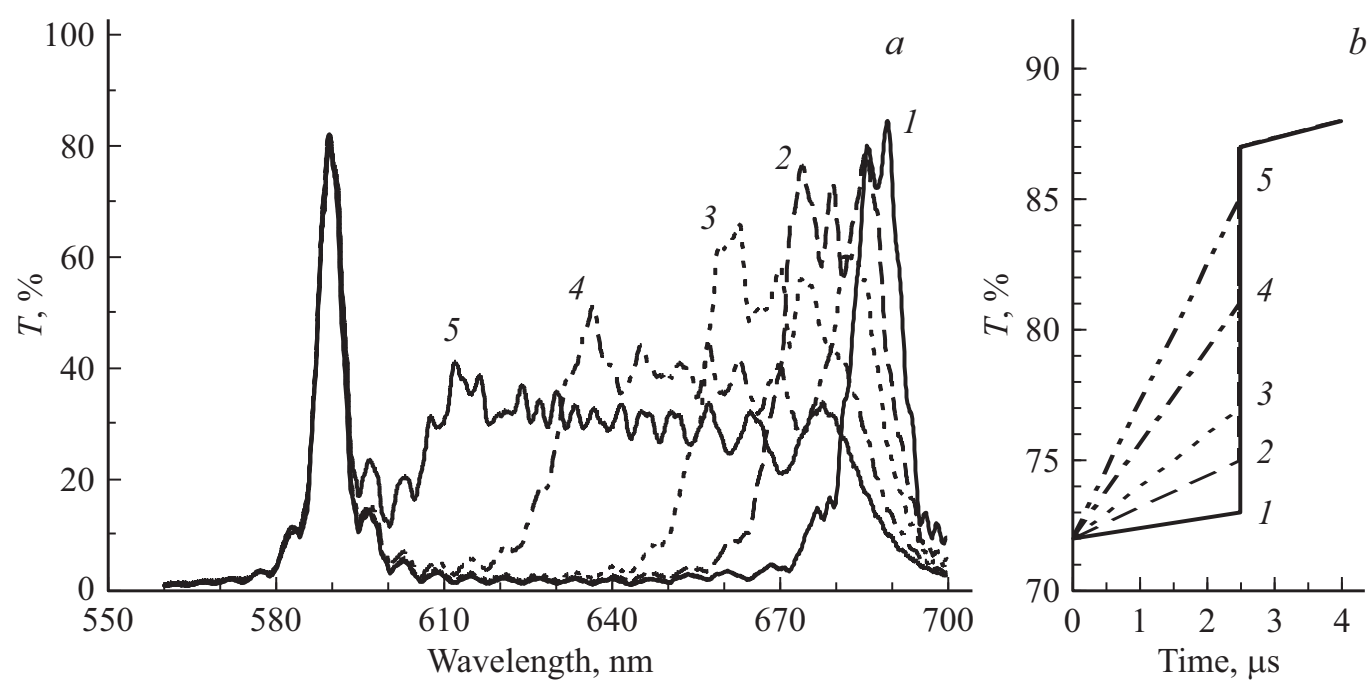

Рис. 5. Синтез асимметричного профиля функции пропускания $(a)$ путем изменения временной зависимости $f(t)$. Справа $(b)-$ формы одного периода пилообразной модуляции (от 72 до $88 \mathrm{MHz}$ за $4 \mu \mathrm{s}$ ).

окон их крылья соединяются, так что зона непропускания в межоконном промежутке постепенно исчезает (просветляется).

На рис. 5, а приведен пример выборочного влияния на разные окна двухоконной функции: ширина одного окна увеличивается вплоть до смыкания со вторым, неизменным окном. В данном примере второе окно, изначально находившееся на краю диапазона, расширяется одновременно со сдвигом своего центра, что иллюстрируется видом временных частотных профилей управляющего ультразвукового сигнала (рис. $5, b$ ). При слиянии окон образуется зона пропускания, покрывающая заданный диапазон спектра и имеющая локальные особенности: пик на длине волны $590 \mathrm{~nm}$ и провал (пониженное пропускание) на $600 \mathrm{~nm}$.

Все синтезированные функции имеют заметную неоднородность в виде осцилляций, что связанно с особенностями функции пропускания АО фильтра. В приближении однократного рассеяния эта функция представляет собой фурье-образ пространственного распределения амплитуды акустического поля $S$ вдоль направления $x$ падающего светового луча

$$
T(v)=\int S(x) \exp \left[2 \pi i\left(v-v_{f}\right) x\right] d x
$$

где $v_{f}$ - оптическая пространственная частота, определяющая положение окна пропускания АО фильтра и определяемая частотой ультразвукового сигнала $v_{f} \sim f$. В связи с тем, что акустическое поле на границах АО ячейки не равно нулю, имеет место обрыв функции $S$ на краях диапазона интегрирования, что вызывает осцилляции ее фурье-образа („явление Гиббса“). Дополнительные осцилляции вызывают и другие разрывы функции $S$, например, резкое изменение частоты в приведенных диаграммах.

\section{Заключение}

Представленные результаты синтеза функций пропускания разного типа: многооконных, с варьируемым положением и шириной окон, в том числе асимметричной формы, демонстрируют принципиальную возможность создания функций пропускания сложного вида. Метод экспресс-регистрации, основанный на корреляционной спектроскопии, в том числе для задач мониторинга с движущегося носителя или в быстро меняющейся обстановке, может быть реализован на основе акустооптических фильтров с цифровым синтезом управляющего высокочастотного сигнала.

Полученные результаты могут быть использованы в различных методах анализа, основанных на управлении шириной окна пропускания фильтра. В частности, в работе [11] предложен метод выравнивания чувствительности АО системы регистрации изображений, основанный на детерминированном изменении ширины окна пропускания при перестройке по спектру. В частности, такой подход может быть целесообразным в тех случаях, когда резервы управления мощностью возбуждаемого ультразвука исчерпаны или ограничены. В этом случае может оказаться необходимым не просто уширять окно пропускания, но и придавать ему асимметричную форму.

\section{Благодарности}

Авторы благодарят В.Н. Шорина за помощь в проведении экспериментов и Л.И. Мазур за помощь в подготовке $\mathrm{AO}$ ячейки.

\section{Конфликт интересов}

Авторы заявляют, что у них нет конфликта интересов. 


\section{Список литературы}

[1] Колесников С.А., Колесникова Е.С., Писаревский Ю.В., Турутин Ю.А. // Кристаллография. 2011. Т. 56. № 1. C. 56-60; Kolesnikov S.A., Kolesnikova E.S., Pisarevsky Yu.V., Turutin Yu.A. // Crystallography Reports. 2011. V. 56. N 1. P. $52-56$.

[2] Писаревский Ю.В., Колесников С.А., Колесникова Е.С., Турутин Ю.А., Конопелько Л.А., Шор Н.Б. // Доклады Академии наук. 2016. Т. 467. № 4. С. 405; Pisarevsky Yu.V., Kolesnikov S.A., Kolesnikova E.S., Turutin Yu.A., Konopelko L.A., Shor N.B. // Doklady Physics. 2016. V. 61. N 4. P. $160-163$.

[3] Пожсар В.Э., Пустовойт В.И. // Известия РАН. Сер. физ. 2015. T. 79. № 10. C. 1375; Pozhar V.E., Pustovoit V.I. // Bull. Russian Academy of Sciences. Physics. V. 79. N 10. P. 1221.

[4] Мазур М.М., Пожсар В.Э. // Измерительная техника. 2015. № 9. C. 29; Mazur M.M., Pozhar V.E. // Optophysical Measurements (Measurement Techniques). 2015. V. 58. N 9. P. 982. doi 10.1007/s11018-015-0829-5

[5] Мазур М.М., Пожар В.Э., Шорин В.Н. // Журн. аналитической химии. 1998. Т. 53. № 9. С. 996; Мazur M.M., Pozhar V.E., Shorin V.N. // J. Analytical Chemistry. 1998. V. 53. N 9. P. 876-878.

[6] Fadeyev A.V., Pozhar V.E., Pustovoit V.I. // SPIE Proc. 2013. V. 8890 . P. 88900 H. doi $10.1117 / 12.2029128$

[7] Проклов В.В., Бышевский-Конопко О.А., Филатов А.Л. // Письма ЖТФ. 2015. Т. 41. № 20. С. 37; Proklov V.V., Byshevski-Konopko O.A., Filatov A.L. // Techn. Phys. Lett. 2015. V. 41. N 10. P. 987.

[8] Pozhar V.E., Pustovoit V.I., Bereza S. // IEEE Ultrasonics Symposium New York, Oct. 28-31, 2007. Proc., P. 829.

[9] Магдич Л.Н., Молчанов В.Я., Пономарева И.П. // Опт. и спектр. 1984. Т. 56. № 4. С. 736

[10] Палавандишвили Л.С., Пожсар В.Э., Пустовойт В.И., Шорин В.Н. // XIII Всес. конф. по акустоэлектронике и квантовой акустике. (Черновцы, 8-10 окт. 1986). Тез. докл. Ч. І. С. 229-230. Киев: АН СССР.

[11] Мазур М.М., Судденок Ю.А., Шорин В.Н. // Письма в ЖТФ. 2014. Т. 40. В. 4. C. 56; Mazur M.M., Suddenok Y.A., Shorin V.N. // Techn. Phys. Lett. 2014. V. 40. N 2. C. 167.

[12] Мазур М.М., Мазур Л.И., Судденок Ю.А., Шорин В.Н. // Оптика и спектр. 2018. Т. 125. В. 4. С. 272; Mazur M.M., Mazur L.I., Suddenok Yu.A., Chorin V.N. // Opt. Spectrosc. 2018. V. 125. N 4. P. 272.

[13] Мазур М.М., Пустовойт В.И., Судденок Ю.А., Шорин В.Н. // Физические основы приборостроения. 2018. T. 7. № 2 (28). C. 20-37. doi 10.25210/jfop-1802.020037; Mazur M.M., Pustovoit V.I., Suddenok Yu.A., Chorin V.N. // Physical Bases of Instrumentation. 2018. V. 7. N 2. P. 20.

[14] Pozhar V.E., Mazur M.M., Machikhin A.S. // Proc. SPIE. 2018. V. 10815. P. 108151I. doi 10.1117/12.2502414

[15] Yushkov K.B., Makarov O.Yu., Molchanov V.Ya. // Optics Lett. 2019. V. 44. N 6. P. 1500. 